xisbegehungen an“, so Kriminaloberkommissar Rainer Both, Landespolizei Saarland. Man berate die Mediziner, wie sie sich besser gegen Einbrüche, aber auch gegen gewaltsame Übergriffe schützen können. „Dazu gehört, dass gefährliche Gegenstände nicht im Griffund Sichtbereich der Patienten liegen sollten“, so Both. Ein Thema sei auch das Verlassen des Gebäudes. So könne man dafür sorgen, dass der Weg bis zum Parkplatz gut ausgeleuchtet ist oder Absprachen treffen, dass der letzte Kollege die Praxis nie allein verlässt. Die Hauptrolle bei schweren Gewalttaten spiele die von Hoffmann erwähnte kalte Aggression. „Sie ist fokussiert, ruhig, strategisch geplant und die Emotionen sind ausgeschaltet", erläuterte Both. „Dabei funktioniert dann keine psychologische Deeskalation." Kalte Aggression baue sich meist in mehreren Stufen auf - von der Fokussierung über Drohungen bis hin zu Probehandlungen und Vorbereitungen wie der Beschaffung von Waffen. „Zeigt sich ein erhöhtes Risikopotenzial bei einem $\mathrm{Pa}$ tienten, dann ist ab einem bestimmten Punkt ein Netzwerk aus Polizei, Psychiater und Experten für Bedrohungsmanagement erforderlich.“

Andreas Kindel
Hier steht eine Anzeige. Springer

\title{
„Es muss Spaß machen, bei Ihnen zu arbeiten!"
}

Eine zeitgemäße Führungskultur ist eine wichtige Voraussetzung dafür, dass sich eine Praxis erfolgreich entwickelt. Das hängt unter anderem damit zusammen, dass in den meisten Arztpraxen die Ausgaben für Personal mit $40 \%$ oder mehr der größte Kostenblock sind. Aber auch die Zufriedenheit der Patienten ist gebunden an die Motivation des Praxisteams - seien dies MFA oder angestellte Ärzte. Den Zusammenhang zwischen diesen beiden Erfolgsfaktoren einer Praxis verdeutlichte der Fachanwalt für Arbeitsrecht Kai Höppner kürzlich beim Seminar „Personalführung und Mitarbeiterauswahl in der Praxis" in Braunschweig. Veranstalter waren die Deutsche Apotheker- und Ärztebank (apoBank) und das Institut für Wirtschaft und Praxis (IWP) in Kooperation mit der KV Niedersachsen. „Es muss Spaß machen, bei Ihnen zu arbeiten“, so der Appell Höppners. Das komme auch bei den Patienten an, es spreche sich herum, es mache sich bemerkbar bei der Suche nach neuen Mitarbeitern und „bestenfalls merken Sie das dann auch, wenn Sie auf Ihre Umsätze schauen", sagte der Arbeitsrechtler.

Zu einer zeitgemäßen Führungskultur gehört laut Höppner vor allem die kooperative Zusammenarbeit mit den Mitarbeitern. Es sei wichtig, MFA und angestellten Ärzten Freiräume zu lassen, damit sie ihre Arbeit selbstständig machen und sich dabei wenigstens teilweise selbst entfalten können. Zu koppeln sei diese Freiheit aber immer mit konkreten Zielen, auf die die Mitarbeiter im Laufe des Jahres zusteuern können und die dann in einem regelmäßig durchgeführten Gespräch zum Jahresende oder -beginn nachgehalten werden können. Solche „Kommunikationsrituale“ wie Mitarbeitergespräche, so Höppner, seien auch wichtig, um festzustellen, wie zufrieden die Mitarbeiter mit ihrer Situation sind. Darüber hinaus seien regelmäßige Teamsitzungen in diesem Zusammenhang von Bedeutung. Dabei sind aktuelle Informationen zur Praxisentwicklung, etwa zur Erweiterung des Praxisspektrums, ebenso anzusprechen wie Fehler und Konflikte. Fehler sind immer als Chance zu sehen, aus ihnen zu lernen und damit zukünftige Fehler zu vermeiden. Nur so kann Offenheit erreicht werden und Fehler werden nicht unter den Teppich gekehrt.

$\mathrm{Zu}$ den zehn Geboten einer zeitgemäßen Mitarbeiterführung gehöre auch, dass die Praxisinhaber bereit seien, ein kritisches Feedback ernst zu nehmen und darauf zu reagieren, sagte Höppner. Nicht zuletzt motiviere die Wertschätzung guter Arbeit von Mitarbeitern dazu, sich auch in Zukunft ins Zeug zu legen.

Hauke Gerlof 